\title{
Acción de repetición en Colombia: ¿Eficaz o Ineficaz?
}

Semillero de investigación IUS EX NUNC Samir Aljach Rayo, Juan David Gil Ruíz, Edwin Alirio Trujillo Cerquera, Oscar Humberto Urueña Medina, Julian Camilo Gutiérrez Trujillo, Luisa Fernanda Gutiérrez Hernández, Lina María Ramírez Urazán, Eliana Melissa Zambrano Artunduaga, Heidy Lorena Sánchez Castillo.

Docente Tutor - Dr. José Hildebrán Perdomo Grupo Conciencia Jurídica / Universidad Surcolombiana

Articulo recibido: 20/09/2009 Aprobado: 03/11/2009

\section{RESUMEN}

Este trabajo producto de los resultados y del análisis de la Investigación desarrollada entre 2008 y 2009 , pretende definir los factores que inciden en la ineficacia de la acción de repetición, acción que fue constituida como herramienta del derecho administrativo para reponer al erario público, el dinero pagado por condenas al Estado, con conductas dolosas o gravemente culposas de sus agentes. Fallas en su procedimiento, en la presentación y desarrollo del debate probatorio, vacíos sustanciales en la norma, e incidencia de la cultura a falta de dolientes al Estado, son algunos de los factores identificados en la investigación que determinan la falta de eficacia y el no cumplimiento de la finalidad de la Acción de repetición, aspectos que se abordan en el presente estudio.

\section{PALABRAS CLAVE}

Acción de Repetición, Culpa Grave, Dolo, funcionarios públicos, requisitos de la Acción, moralidad, indemnización, condenas al Estado.

\section{ABSTRACT}

This presentation of the results and analysis of our research work, we can identify factors that influence the ineffectiveness of the action for indemnity, an action that was established as a tool of administrative law to the public purse to replenish the money paid because of convictions to the state, when the misconduct or serious negligence of their agents. Failures in its procedure, the presentation and the discussion of evidence, substantial gaps in the standard, and impact of culture in the absence of mourners to the State, are among the factors identified in our investigation determined that the lack of effectiveness and not fulfillment of the purpose of the action replay, both of which are addressed in this study.

\section{KEYWORDS}

Action, Gross negligence, Public Officials, Requirements of the Action, Morality, Compensation, Convictions to the State Exchequer.

\section{INTRODUCCION}

El Estado colombiano se ve enfrentado a diario con un sin número de requerimientos económicos, ya sea para suplir las necesidades básicas de sus ciudadanos o para atender los pagos que demanda administrar el Estado. Para el buen funcionamiento de la administración pública, se implementa toda una infraestructura de personal, funcionarios públicos y particulares con funciones públicas, con el objetivo de 
cumplir y alcanzar los fines del Estado. Es así que al enfrentar dichos compromisos representados en cifras numéricas, se observa con mucha preocupación que el Estado gasta gran parte de su presupuesto en el pago de las condenas en contra de sus entidades por errores de sus agentes, siendo esto una problemática social y de economía pública, pues del pago que hacemos con nuestras contribuciones es que se sostiene en su mayoria la infraestructura de nuestro Estado. Estos recursos que bien podrían ser destinados a satisfacer necesidades básicas de la comunidad, recaigan en el pago de condenas.

De ésta manera nuestro ordenamiento jurídico, implementó una herramienta de derecho administrativo, para que se propendiera por la defensa del patrimonio estatal, en razón de perseguir a titulo de indemnización el reembolso de los pagos hechos por condenas ocasionadas por conductas dolosas o gravemente culposas de los agentes del Estado a las entidades que sufrieron el menoscabo en su patrimonio.

La Ley 678 de 2001 surgió como reglamentación del inciso 2 del artículo 90 de la constitución política, ya que allí el constituyente primario, incluyó la obligación de repetir contra los agentes del Estado que ocasionaran un daño antijurídico a la sociedad. De esta manera se consignaron en dicha Ley, los fundamentos sustanciales y procedimentales de la acción de repetición. Pero la discusión jurídica y de la cual se desprende el problema de investigación, recae al observar que dicha ley nació con la finalidad de perseguir en forma de indemnización el pago del valor de la condena sufrida por el Estado por parte del agente que dio lugar a ella con su conducta, finalidad que a nuestro juicio es altamente cuestionable. Es así que nuestro ánimo investigativo nos ha conducido a identificar acerca de la eficacia o ineficacia de la Acción de Repetición, esto con el fin de determinar si se está cumpliendo a cabalidad la finalidad para la cual fue creada.

De esta manera fijamos como objetivo de la presente investigación el identificar los factores que podrían determinar la eficacia o la ineficacia en la aplicación de la acción de repetición en los distritos judiciales de Neiva, Florencia, Popayán, Ibagué y Bogotá, desde la entrada en vigencia de la Ley 678 de 2001.

\section{METODOLOGÍA}

El enfoque metodológico que se implementa en la presente investigación, fue el de la investigación cuantitativa descriptiva, puesto que comprendió la descripción, registro, análisis e interpretación de la situación actual en la aplicación de la Acción de Repetición en la Jurisdicción Administrativa y Contraloría General de la República y sus delegadas, en el Huila, Tolima, Florencia, Caquetá y Bogotá; De cuyo resultados se extrajeron las conclusiones más dominantes de la problemática investigada.

La investigación fue aplicada mediante un instrumento con el cual se extrajo los datos referentes a determinar los factores de la eficacia o ineficacia de la Acción de Repetición sobre la totalidad de los procesos que versaban sobre acción de repetición, en los Distrito Administrativos del Huila, Caquetá, Cauca, Tolima y una Muestra en el Distrito Administrativo de Bogotá y el Consejo de Estado.

\section{MARCO TEÓRICO}

Las primeras nociones referentes a la acción de repetición las encontramos desde 1976 con el Estatuto Contractual de la Nación Decretoley 150 , en el que se incluyó una responsabilidad patrimonial solidaria por parte de los agentes públicos, y la entidad estatal, pero solo en lo concerniente al tema de contratación ${ }^{1}$.

Posteriormente, el Decreto-ley 222 de 1983 estableció la responsabilidad civil de los empleados oficiales por los perjuicios que causaran a las entidades, originados en la celebración de contratos sin el cumplimiento de los requisitos y formalidades legales. La acción respectiva podía

${ }^{1}$ Sentencia del 31 de Mayo de 2007. Radicado: 21.101. Actor: Nación-Contraloría General de la República.C.P. Enrique Gil Botero. 
ser instaurada por el representante legal de la entidad contratante o por la Procuraduria General dolosa o gravemente culposa del funcionario, y ésta a su vez haya quedado ejecutoriada y cancelada para impetrar la repetición. Es importante aclarar dicha característica citando como ejemplo la siguiente pronunciamiento realizado por el Honorable Consejo de Estado en el cual dijo:

"Si un servidor público, con un acto suyo doloso o gravemente culposo, que perjudica a un particular, ocasiona una condena al Estado, incurre para con éste en responsabilidad civil, que debe ser judicialmente declarada. Pero si dicho servidor, en ejercicio de sus competencias para administrar o custodiar bienes o fondos, causa su pérdida, incurre en responsabilidad fiscal, cuyo pronunciamiento está reservado a la Contraloría."

5. Es Secundaria; Debido a que es una consecuencia de la declaración de la responsabilidad del Estado, ya sea por sentencia, por conciliación o cualquier medio de terminación de conflicto.

6. Es Patrimonial: Tiene por objeto la recuperación a modo de indemnización por parte del funcionario público el valor pagado por la entidad en razón a la condena en que se declaró la responsabilidad estatal.

El legislador estableció que la finalidad de la acción de repetición se encaminaba a garantizar los principios de moralidad y eficiencia de la función pública, sin perjuicio de los fines retributivo y preventivo inherentes a ella $a^{6}$ con la cual se pretende generar un efecto preventivo frente a los funcionarios públicos. Para que dicha finalidad se materialice, el Consejo de Estado en su Sección Tercera $^{7}$, ha puntualizado los siguientes requisitos para que sea procedente la acción:

1. Que una entidad pública haya sido condenada, por la jurisdicción contencioso administrativa, a reparar los daños antjurídicos causados a un particular.

${ }^{2}$ Véase Sentencias del Consejo de Estado, Sección Tercera. Radicado: 27.148; 22.101; 19.726; 22.527, entre otras.

${ }^{3}$ Sentencia de 11 de septiembre de 2003. Radicado: D-4477. Actor: William León M. Magistrado.Ponente. Jaime Araujo Rentería.

“CARTILLA INSTRUCTIVA DE ACCIÓN DE REPETICIÓN Y LLAMAMIENTO EN GARANTÍA. Programa Presidencial de Lucha Contra la CorrupciónMinisterio de Justicia y del Derecho: Dirección de Defensa Judicial de la Nación. Pág. 8. 
2. Que se haya establecido que el daño antijurídico fue consecuencia de la conducta dolosa 0 gravemente culposa de un agente 0 antiguo ex agente público.

3. Que la entidad condenada haya pagado la suma de dinero determinada por el juez en su sentencia.

Una vez definido el criterio de investigación, y al haber elaborado una descripción respecto a la problemática abordada, nos damos en la tarea de resaltar los hallazgos y la información que arrojó el estudio de los procesos de Acción de Repetición

\section{RESULTADOS} en los Distritos Administrativos del Huila, Caquetá, Tolima, Cauca y la muestra en el Distrito Administrativo de Cundinamarca y el Honorable Consejo de Estado, al igual que las entrevistas aplicadas a los entes de control en especial a las Contralorías Delegadas de los anteriores Departamentos.

\subsection{Consolidado de procesos de Acción de Repetición investigados.}

Una vez identificado el universo, se determina que en los Despachos de los Juzgados y Tribunales de lo contencioso Administrativo, los procesos que versaban a cerca de la Acción de Repetición son muy pocos, comparado con otras acciones como las de Nulidad y Restablecimiento del Derecho y Reparación que las superan ampliamente en número. Es así que en todo el universo, se encontró que se han impetrado 455 procesos por Acción de Repetición distribuidos, y en su mayoría se encuentran en tramite, tal como nos lo muestra la ilustración.

${ }^{5}$ Sentencia del 26 de julio de 2001, Exp. 6620, M. P. Camilo Arciniegas Andrade. 
Cuadro 1: Consolidado de Procesos de Acción de Repetición Investigados.

$\begin{array}{lcccccccccccc}\text { CATEGORIAS } & \begin{array}{c}\text { BTA } \\ \text { C.E }\end{array} & \begin{array}{c}\text { BTA } \\ \text { TR }\end{array} & \begin{array}{c}\text { BTA } \\ \text { JUZ }\end{array} & \begin{array}{c}\text { FLOR/CIA } \\ \text { TR }\end{array} & \begin{array}{c}\text { FLOR/CIA } \\ \text { JZG }\end{array} & \begin{array}{c}\text { IBAG } \\ \text { TR }\end{array} & \begin{array}{c}\text { IBAG } \\ \text { JUZ }\end{array} & \begin{array}{c}\text { PYAN } \\ \text { TR }\end{array} & \begin{array}{c}\text { PYAN } \\ \text { JUZ }\end{array} & \begin{array}{c}\text { NEV } \\ \text { TR }\end{array} & \begin{array}{c}\text { NEV } \\ \text { JUZ }\end{array} & \text { TOTAL } \\ \text { FALLADO5 } & \mathbf{2 8} & \mathbf{1 8} & \mathbf{0} & \mathbf{0} & \mathbf{2} & \mathbf{1 2} & \mathbf{3} & \mathbf{3} & \mathbf{1} & \mathbf{3} & \mathbf{6} & \mathbf{7 6} \\ \text { ENTRAMITE } & \mathbf{8} & \mathbf{5 1} & \mathbf{3 2} & \mathbf{2} & \mathbf{1 7} & \mathbf{3 1} & \mathbf{8 6} & \mathbf{1 4} & \mathbf{6 2} & \mathbf{2 0} & 56 & \mathbf{3 7 9} \\ \text { TOTAL } & \mathbf{3 6} & \mathbf{6 9} & \mathbf{3 2} & \mathbf{2} & \mathbf{1 9} & \mathbf{4 3} & \mathbf{8 9} & \mathbf{1 7} & \mathbf{6 3} & \mathbf{2 3} & \mathbf{6 2} & \mathbf{4 5 5}\end{array}$

\subsection{Cuantía de las pretensiones de la Acción de Repetición}

Al momento de analizar la cuantía de las pretensiones en las demandas deAcción de Repetición, nos arrojó como resultado que en el $58,68 \%$ de los procesos de repetición revisados, corresponden a la mayor cuantía, el $30,54 \%$ lo constituyen los procesos de menor y encontramos un una minoría del $9,45 \%$ de mínima cuantía, lo que nos estaría indicando que pese a las pocas demandas instauradas por las entidades públicas, no se está haciendo un recuperación representativa, en comparación al pago que hace el Estado por condenas en su contra.

Cuadro 2: Cuantía de las Pretensiones de la Acción de Repetición

$\begin{array}{lcccccccccccc}\text { CUANTIA DE } & \text { C. E. } & \text { TRIB } & \text { JUZ } & \text { TRIB } & \text { JUZ } & \text { TRIB } & \text { JUZG } & \text { TRIB } & \text { JUZG } & \text { TRIB } & \text { JUZG } \\ \text { LA ACCIÓN } & & \text { BTA } & \text { BTA } & \text { FLOR } & \text { FLOR } & \text { IBAG } & \text { IBAG } & \text { POPAY } & \text { POPAY } & \text { NEIVA } & \text { NEIVA } & \text { TOTAL } \\ \text { MINIMA CUANTIA } & 0 & 3 & 5 & 0 & 1 & 3 & 12 & 3 & 5 & 1 & 10 & 43 \\ \text { MENOR CUANTIA } & 1 & 14 & 10 & 0 & 7 & 12 & 30 & 2 & 32 & 9 & 22 & 139 \\ \text { MAYOR CUANTIA } & 29 & 52 & 17 & 2 & 11 & 28 & 47 & 12 & 26 & 13 & 30 & 267 \\ \text { NO HAY DATO } & 6 & 0 & 0 & 0 & 0 & 0 & 0 & 0 & 0 & 0 & 0 & 6 \\ \text { TOTAL } & 36 & 69 & 32 & 2 & 19 & 43 & 89 & 17 & 63 & 23 & 62 & 455\end{array}$

\subsection{Origen de la Acción de Repetición.}

Observamos que las condenas profenidas en contra del Estado tienen su fuente en una alta proporción $(35,16 \%)$ en las Acciones de Nulidad y Restablecimiento del Derecho, pero específicamente y con notoria superionidad $(60,62 \%)$ derivadas de la desvinculación de servidores públicos en forma irregular (Grafica 3.1). Le siguen las condenas como consecuencia de acciones de Reparación Directa (23,95\%); luego por conciliaciones debidamente aprobadas por los órganos competentes (14,72\%); renglón seguido las condenas con ocasión de relaciones laborales (12,74\%); finalizando con los Procesos Ejecutivos y Acciones Contractuales, con un (4,61\%) y otros procesos en menor cantidad en un $(4,17 \%)$. Se determinó que el Distrito Administrativo del Tolima, es el que presenta en mayor proporción demandas de Acción de Repetición por condenas en procesos de nulidad y restablecimiento del derecho.

Cuadro 3: Origen de la Acción de Repetición.

\begin{tabular}{|c|c|c|c|c|c|c|c|c|c|c|c|c|}
\hline TIPO DE ACCIÓN & C. E. & $\begin{array}{l}\text { TRIB } \\
\text { BTA }\end{array}$ & $\begin{array}{l}\text { JUZ } \\
\text { BTA }\end{array}$ & $\begin{array}{l}\text { TRIB } \\
\text { FLOR }\end{array}$ & $\begin{array}{c}\text { JUZ } \\
\text { FLOR }\end{array}$ & $\begin{array}{l}\text { TRIB } \\
\text { IBAG }\end{array}$ & $\begin{array}{l}\text { JUZG } \\
\text { IBAG }\end{array}$ & $\begin{array}{c}\text { TRIB } \\
\text { POPAY }\end{array}$ & $\begin{array}{c}\text { JUZG } \\
\text { POPAY }\end{array}$ & $\begin{array}{c}\text { TRIB } \\
\text { NEIVA }\end{array}$ & $\begin{array}{c}\text { JUZG } \\
\text { NEIVA }\end{array}$ & TOTAL \\
\hline Ny R.D. & 17 & 28 & 4 & 0 & 6 & 18 & 33 & 2 & 17 & 14 & 21 & 160 \\
\hline R.D & 3 & 16 & 16 & 1 & 10 & 14 & 15 & 7 & 10 & 5 & 12 & 109 \\
\hline CONCILIACION & 2 & 9 & 3 & 0 & 2 & 4 & 10 & 7 & 16 & 1 & 13 & 67 \\
\hline ACC. CONT. & 2 & 8 & 4 & 0 & 0 & 2 & 3 & 0 & 2 & 0 & 0 & 21 \\
\hline ACC. EJEC & 0 & 2 & 0 & 0 & 0 & 3 & 7 & 0 & 3 & 0 & 6 & 21 \\
\hline PROC ORD. LAB. & 2 & 3 & 2 & 1 & 1 & 1 & 19 & 1 & 15 & 3 & 10 & 58 \\
\hline OTRO & 10 & 3 & 3 & 0 & 0 & 1 & 2 & 0 & 0 & 0 & 0 & 19 \\
\hline TOTAL & 36 & 69 & 32 & 2 & 19 & 43 & 89 & 17 & 63 & 23 & 62 & 455 \\
\hline
\end{tabular}


El fin último de la Acción de Repetición, de propender por una moral administrativa y un buen desempeño de las funciones de sus servidores públicos, es notoriamente cuestionable a la luz de estos hallazgos, ya que la principal fuente de litigios contenciosos administrativos se derivan de procesos de nulidad y restablecimiento del derecho, y para el caso concreto, de tal acción es la que se desprende la mayor cantidad de procesos de repetición derivada de conductas que a la luz del análisis jurídico y sociológico surgen del mal proceder del funcionario a cargo, ya que la desvinculaciones o declaraciones de insubsistencia, la responsabilidad extracontractual o el simple hecho de emitir un acto administrativo que cause perjuicio, son la constante en la sociedad colombiana y es que se refleja en estos resultados.

Cuadro 3.1: Subcategorías, conductas reincidentes que dan origen a la Acción de Repetición.

$\begin{array}{ccc}\text { ACCIÓN } & \text { HECHOS QUE ORIGINARON LA REPETICIÓN } & \text { TOTAL } \\ \text { NULIDAD Y RESTABLECIMIENTO } & \text { Desvinculación O Decl. de insubsistencia } & 129 \\ \text { DEL DERECHO } & \text { Acto adtivo. que causo perjuicio } & 31 \\ \text { REPARACIÓN DIRECTA } & \text { Resp. Extracontractual del Estado } & 83 \\ & \text { Acto adtivo. que causo perjuicio } & 26 \\ & \text { Desvinculación administrativa } & 17 \\ \text { CONCILIACIÓN } & \text { Acto adtivo. que causo perjuicio } & 3 \\ & \text { Resp. Extracontractual del Estado } & 20 \\ \text { ACCIÓN CONTRACTUAL } & \text { Accion Originada de Contrato } & 12 \\ & \text { Rel. Individ. Trabajo (proc. Ord.laboral) } & 15 \\ \text { PROC. ORD. LABORAL } & \text { Relación Contractual } & 21 \\ & \text { Acc.ejecutiva } & 21 \\ \text { OTRO } & \text { Incumplim. De Obligaciones Laborales } & 29 \\ & \text { Relac. Laboral Originada En Cto Ind. Trabajo } & 29 \\ & \text { Procesos Ord. Civil, Acc. de Grupo, de Tutela, Popular, entre otros } & 11 \\ & \text { No hay dato } & 8 \\ & \text { TOTAL }\end{array}$

\subsection{Incidencia de las entidades públicas al formular Acción de Repetición.}

Al determinar el nivel de la entidad demandante, encontramos que en un $50,98 \%$ de las acciones halladas, las entidades de orden municipal son quienes impetran en su mayoria dicho litigio, seguida de las entidades de orden nacional en un $27,91 \%$, restando tan solo un $18,02 \%$ al orden departamental y un $2,64 \%$ a las entidades Autónomas Constitucionales. Cabe advertir que pese a que las entidades de orden municipal son las que más inician procesos por Acciones de Repetición, los valores a recuperar no son muy representativos, contrario sensu a las entidades de orden nacional, quienes lideran los listados por el monto del valor en sus condenas, y de las cuales no se inician este tipo de procesos para recuperar estos dineros públicos, verbigracia, el Ministerio de Agricultura y Desarrollo Rural, que mediante contestación de derecho de petición enviado por el semillero de investigación informó que en la vigencia del 2007, pagó por concepto de sentencias y conciliaciones el valor de CATORCE MIL CUATROCIENTOS OCHENTA Y UN MIL MILLONES DE PESOS M/C ( $\$ 14.481 .000 .000,00)$, de los cuales el Ministerio no ha iniciado ninguna acción de repetición hasta el momento.

Cuadro 4: Nivel de la Entidad Demandante en Acción de Repetición.

$\begin{array}{ccc}\text { NIVEL DE LA ENTIDAD DEMANDANTE } & \mathbf{N}^{\bullet} \text { PROCESOS } & \text { PORCENTANE } \\ \text { NACIONAL } & 127 & \% \\ \text { DEPARTAMENTAL } & 82 & 27,91 \% \\ \text { MUNICIPAL } & 232 & 18,03 \% \\ \text { AUTÓNOMO CONSTITUCIONAL } & 12 & 50,98 \% \\ \text { NO HAY DATO } & 2 & 2,64 \% \\ \text { TOTAL } & 455 & 0,44 \% \\ \end{array}$




\section{CONCIUSIONES}

En primer lugar se puede concluir que la Acción de Repetición no es un mecanismo eficaz, porque la ley que la reglamenta presenta diferentes vacios que obstruyen o impiden la cabal aplicación de este mecanismo jurídico; sumado a lo anterior la falta de compromiso, de los representantes legales de las entidades públicas, en hacer uso de este instrumento, producto de los intereses personales, económicos y politiqueros que constituyen corrupción administrativa. En igual sentido, algunos de los representantes legales de las entidades públicas ignoran la existencia de dicha acción, son negligentes e incurren en dilaciones dejando prescribir los términos para la iniciación de la acción o abandonando a su suerte el debate probatorio lo que genera en la mayoría de ocasiones fallos absolutorios al agente responsable; por último, la omisión en el ejercicio de las competencias asignadas a los organismos de control fiscal y del ministerio público, quienes tienen la potestad de impetrar esta acción, en ausencia de ejercicio por parte de las entidades públicas directamente afectadas.

Como bien lo indican los resultados, este instrumento jurídico, no se está aplicando de forma eficaz, no solo porque se desconoce su realización, sino porque en la mayoría de los casos, el representante legal (quien debe iniciar el procedimiento) lo hace finalmente solo con el objeto de cumplir un requisito legal ya que asi se le ordena y no con el fin de recuperar el dinero pagado por condenas a la entidad.

La Ley 678 de 2001 en su aplicación sustancial y procesal no es la única causante de esta falta de eficacia, pues como se ha anotado en varias ocasiones, la mayor responsabilidad recae en la misma administración, y desafortunadamente al Estado le falta quien se compadezca de su situación, pues como lo titula la norma y la
Constitución lo que se pretende con dichos procedimientos es fortalecer la moralidad administrativa y reducir los índices de corrupción del Estado. Sin embargo la Acción de Repetición solo se ha convertido en un formalismo y no una herramienta para reponer lo que se ha perdido por culpa de sus mismos administradores.

Al analizar los fundamentos procesales y probatorios de la acción, encontramos que aunque el Honorable Consejo de Estado ha fijado en varias de sus providencias los requisitos para que prospere el ejercicio de la Acción de Repetición, estos son poco aplicados y es por eso que en los pocos procesos iniciados, al final se deniegan las pretensiones de la demanda por falta de cumplimiento de estos requisitos.

Unos de los obstáculos que se encuentra el juez a la hora de estudiar los casos de reposición es el de determinar sila conducta del agente fue dolosa o gravemente culposa, ya que la ley no lo hizo de manera clara, y es por ellos que es el juez mediante la presentación de las pruebas quien debe analizar y determinar si se actuó en contra de la administración con dolo y/o culpa grave.

Unido al anterior inconveniente, encontramos que las entidades al momento de demandary allegar las pruebas para que se le configure los requisitos de las Acción de repetición y se declare al agente la obligación de pagar a titulo de indemnización lo que la entidad canceló por la condena causada por su conducta, no se tienen en cuenta los presupuestos probatorios fijados por el ordenamiento jurídico y que es reincidentemente resaltado por la jurisprudencia, ya que la entidad en su afán de impetrar la acción, allega en copias simples los documentos que deben ser autenticados para que tenga validez probatoria. Del mismo modo se allegan al proceso pruebas que no tienen la validez y que lo único que se puede esperar es que las pretensiones sean denegadas.

\footnotetext{
"El Honorable Consejo de Estado, Sección Tercera, ha venido realizando últimamente en la mayoría de sus providencias una admonición a los representantes legales de las entidades demandantes y a sus apoderados, para que al momento de acreditar el pago de la condena por parte de la entidad allegue conforme lo recita la norma. El recibo de pago, comprobante de egreso, etc. Autenticado y no en fotocopia simple, ya que este no tiene sustento probatorio dando lugar a que se profiera un fallo absolutorio, y así se perjudique al Estado, con una herramienta que fue creada para su beneficio. Véase las Sentencias: Exp. 22.552; Exp. 27.202; Exp. 31.466; Exp. 27.552; Exp. 26.226; Exp. 19.726, entre otras.
} 
Otro factor que determina la ineficacia de la acción de repetición se presenta en el valor de la condena puesto que en la casi totalidad de los casos esta se vuelve totalmente simbólica, por imposibilidad absoluta de pago del servidor público sancionado.

Bastaría comparar el monto anual de las condenas en acción de repetición, efectivamente pagadas por los servidores públicos condenados, con los gastos administrativos que tienen que asumir las entidades públicas demandantes y la jurisdicción de lo contencioso administrativo en el trámite de las mismas, para concluir que la acción de repetición, desde el punto de vista del objetivo de recuperación del patrimonio público, es totalmente ineficaz e ineficiente.

Lo cierto es que el monto de las condenas en la acción de repetición es elevado. Pero en la práctica se torna impagables, cuando la condena recae en un servidor público de escasos recursos. Una consecuencia es que excluye de por vida a estos servidores de conformidad con el artículo 122 de la Constitución Política, que fue reformado por el artículo primero del Referendo del año 2003. Estos servidores públicos condenados en acción de repetición no pueden inscribirse como candidatos de elección popular, ni ser designados como servidores públicos, ni celebrar contratos con el Estado, hasta tanto no paguen el valor de la condena.

Ahora frente a los requisitos de procedibilidad para hacer efectiva la acción de repetición, se encuentra otro factor más que nos lleva a concluir que esta acción no cumple con el objetivo para el cual fue creada; este requisito tiene que ver con la puesta en marcha de la acción, la cual debe ser a partir del pago efectivo de la indemnización por el estado, lo que presenta como problema ya que en la espera de que el Estado desembolse efectivamente el dinero y que al proceso se aporte prueba del recibo del pago de totalidad del mismo, pueden pasar muchos años, dando lugar a que el funcionario o ex funcionario se insolvente, lo que permite que la acción pierda toda su razón de ser.

Para finalizar creemos que otro factor que determina la ineficacia de la acción tiene que ver con el tema de medidas cautelares, en el sentido de que no existe una protección a la entidad cuando esta solicita dichas medidas, ya que si la entidad solicita las medidas y el fallo no es favorable pues será a ella a la que le acarrearían múltiples sanciones. Esto hace que las entidades se curen en salud y no soliciten medidas. 


\section{BIBLIOGRAFÍA}

- Benavides León, Edgar Arturo (2003). Acción de repetición, Ley 678 de 2001: Comentarios y concordancias. Bogotá, Ediciones Librería del Profesional.

- CARTILLA INSTRUCTIVA DE ACCIÓN DE REPETICIÓN Y LLAMAMIENTO EN GARANTÍA (2005). Programa Presidencial de Lucha Contra la Corrupción-Ministerio de Justicia y del Derecho: Dirección de Defensa Judicial de la Nación.

- Rodríguez Rodríguez, Libardo (2004). Derecho Administrativo Colombiano; México; Porrúa: UNAM, Instituto de Investigaciones Juridicas.

- Sáchica, Luís Carlos (2006). Derecho Constitucional General. Bogotá, Editorial Biblioteca Jurídica Diké.

- Santofimio, Jaime (2008). Tratado de Derecho Administrativo, Tomo I y ll. Bogotá, Editorial Universidad Externado de Colombia.

- Vidal Perdomo, Jaime (2008). Derecho Administrativo. Bogotá, Editorial Temis.

- Álvarez-Correa, Miguel, Corzo M. Lino, Figueroa M, David. (2005). Jueces de paz: una figura de la justicia comunitaria: análisis. Bogotá, Instituto de Estudios del Ministerio Público.

- Cobos Gavala, Rosa. (1989). Jueces de paz en la ordenación jurisdiccional española. Madrid, Ministerio de Justicia.

- Alonso y Colmenares, Alonso. (2007). Práctica de los juzgados de paz de las islas de Cuba y Puerto Rico. Boston, Universidad de Harvard.

- Boaventura de Sousa Santos y Garcia Villegas, Mauricio. Caleidoscopio de las justicias en Colombia. Bogotá, Siglodel Hombre Editores. 


\section{REFERENCIAS LEGALES}

Fundamentos legales

- Decreto-ley 01 de 1984

- Decreto-ley 222 de 1983

- Decreto-ley 150 de 1976

- Ley 80 de 1993

- Ley 270 de 1996 (vigente)

- Ley 446 de 1998

- Ley 678 de 2001

\section{REFERENCIAS JURISPRUDENCIALES}

Corte Constitucional Colombiana

- República de Colombia. Corte Constitucional. Sentencia C-233 de 2002. M.P. Álvaro Tafur Galvis. Exp. D-3704.

- República de Colombia. Corte Constitucional. Sentencia C-414 de 2002. M.P. Manuel José Cepeda. Exp. D-3781.

- República de Colombia. Corte Constitucional. Sentencia C-285 de 2002. M.P. Jaime Córdoba Triviño. Exp. D-3736.

- República de Colombia. Corte Constitucional. Sentencia C-455 de 2002. M.P. Marco Gerardo Monroy Cabra. Exp. D-3826.

- República de Colombia. Corte Constitucional. Sentencia C-309 de 2002. M.P. Jaime Córdoba Triviño. Exp. D-3754.

- República de Colombia. Corte Constitucional. Sentencia C-484 de 2002. M.P. Álvaro Beltrán Sierra. Exp. D-3824; D-3827; D-3812; D-3833, acumulados.

- República de Colombia. Corte Constitucional. Sentencia C-372 de 2002. M.P. Jaime Córdoba Triviño. Exp. D-3770; D-3775.

- República de Colombia. Corte Constitucional. Sentencia C-374 de 2002. M.P. Clara Inés Vargas Hernández. Exp. D-3756; D-3757; D-3763.

- República de Colombia. Corte Constitucional. Sentencia C-378 de 2002. M.P. Marco Gerardo Monroy Cabra. Exp. D-3789.

- República de Colombia. Corte Constitucional. Sentencia C-394 de 2002. M.P. Álvaro Tafur Galvis. Exp. D-3773.

Consejo de Estado.

- Sentencia del 31 de Mayo de 2007. Radicado: 21.101. Actor: Nación-Contraloría General de la República. C.P. Enrique Gil Botero.

- Sentencia de 11 de septiembre de 2003. Radicado: D-4477. Actor: William León M. M.P. Jaime Araujo Renteria.

- Sentencia del 26 de julio de 2001. Exp.6620, M. P. CamiloArciniégas Andrade.

- Sentencia del 5 de Diciembre de 2007. Radicado 27.202; Autor: Instituto de Desarrollo Urbano IDU. C.P. Enrique Gil Botero.

- Sentencia del 4 de Diciembre de 2007. Radicado 27.148; Autor: Instituto de Desarrollo Urbano IDU. C.P. Mauricio Fajardo Gómez. 